\title{
Neural stem/progenitors and glioma stem-like cells have differential sensitivity to chemotherapy
}

\author{
ate
}

Xing Gong, MD

Philip H. Schwartz, PhD

Mark E. Linskey, MD

Daniela A. Bota, MD, $\mathrm{PhD}$

Address correspondence and reprint requests to Dr. Daniela A. Bota, UC Irvine Medical Center, 101 The City Drive South, Shanbrom Hall, Suite 121, Orange, CA 92868 dbota@uci.edu

Editorial, page 1118

Supplemental data at www.neurology.org

\section{ABSTRACT}

Objectives: New data suggest that glioma stem-like cells (GSCs) and neural stem/progenitor cells (NSCs) may share common origins. GSCs drive tumor proliferation and appear to be resistant to classic chemotherapy, while the effects of chemotherapy on NSCs are not well studied. As the role of NSCs in learning and memory is increasingly recognized, we need to identify drugs that reduce neurotoxicity but are still effective against glial tumors.

Methods: We treated 3 human NSC cultures and multiple low- and high-grade GSC cultures with the commonly used agents temozolomide (TMZ) and cisplatin (CIS), and with 2 newer, promising drugs: the proteasome inhibitor bortezomib (BTZ) and the epidermal growth factor receptor tyrosine kinase inhibitor erlotinib (ERL). We measured cell survival, proliferation, cell death induction, and drug resistance markers.

Results: TMZ decreased NSC viability, while minimally affecting GSCs. TMZ induced NSC death, which was partially compensated for by increased proliferation. CIS had similar effects. The NSC's sensitivity to TMZ and CIS correlated with low expression of the multidrug resistance gene ABCG2, but not of MGMT or MSH1/MLH2. BTZ caused an 80\% decrease in GSCs, while minimally affecting NSCs. GSCs had lower proteasome levels and activity after BTZ treatment. ERL treatment also decreased GSC numbers, but not NSC viability, which correlated with low EGFR expression in NSCs compared to GSCs.

Conclusions: Newer chemotherapy agents ERL and BTZ are effective against GSCs yet produce minimal effects on NSCs, while the older drugs TMZ and CIS are more toxic for NSCs than for GSCs. The identification and testing of more selective drugs is clearly warranted. Neurology ${ }^{\circledR}$ 2011;76:1126-1134

\section{GLOSSARY}

BTZ = bortezomib; CIS = cisplatin; ERL = erlotinib; GSC = glioma stem-like cell; $\mathbf{M G M T ~ = ~ m e t h y l - g u a n i n e ~ m e t h y l ~ t r a n s - ~}$ ferase; $\mathbf{N S C}=$ neural stem/progenitor cell; $\mathbf{T M Z}=$ temozolomide.

In the United States, 22,000 people per year are diagnosed with primary brain tumors. ${ }^{1}$ Their quality of life is diminished by treatment-induced deficits of learning and memory, ${ }^{2-4}$ manifested across cognitive domains. Such deficits occur in patients with systemic malignancies who receive chemotherapy but not brain radiation. ${ }^{5}$

Various agents are used to treat brain tumors. Temozolomide (TMZ), a commonly used neuro-oncology drug, and cisplatin (CIS), a second-line glioma treatment, achieve high concentrations in the brain. ${ }^{6,7} \mathrm{TMZ}$ is a DNA-alkylating agent, whereas CIS forms platinum DNA adducts. TMZ resistance is due to high methyl-guanine methyl transferase (MGMT) levels. ${ }^{8}$ Resistance to CIS relates to inactivation of mismatch repair enzymes MLH1 and MSH2 ${ }^{9}$ and overexpression of multidrug resistance proteins, especially ABCG2. ${ }^{10}$ Two additional classes, epidermal growth factor receptor tyrosine kinase inhibitors (erlotinib [ERL]) ${ }^{11,12}$ and the

e-Pub ahead of print on February 23, 2011, at www.neurology.org.

From the Departments of Neurology (X.G., D.A.B.) and Neurological Surgery (M.E.L., D.A.B.), UC Irvine School of Medicine, Orange; Children's Hospital of Orange County Research Institute (P.H.S.), Orange; and Chao Family Comprehensive Cancer Center (M.E.L., D.A.B.), Irvine, CA.

Study funding: Supported in part by start-up funds to D. Bota from the University of California, Irvine, and by Children's Hospital of Orange County through the National Human Neural Stem Cell Resource.

Disclosure: Author disclosures are provided at the end of the article. 
proteasome inhibitors (bortezomib [BTZ]), ${ }^{13}$ have passed preclinical testing, ${ }^{14,15}$ and showed results in small studies.

Stem cell populations are important for both tumor behavior and cognition. A subpopulation of glioblastoma cells - the glioma stem-like cells $(\mathrm{GSCs})^{16}$ — are positive for neural stem/progenitor cells (NSCs) markers. ${ }^{17,18}$ GSCs are resistant to radiation, ${ }^{19}$ and to $\mathrm{TMZ}$, carboplatin, paclitaxel, and etoposide, ${ }^{20}$ but their sensitivity to ERL or BTZ relative to non-GSC tumor components or the NSCs is not yet described. By contrast, NSCs persist throughout adulthood. ${ }^{21}$ Neurogenesis is important for learning ${ }^{22}$ and memory. ${ }^{23}$ Radiation, ${ }^{24}$ and carmustine, CIS, and 5-fluorouracil, are toxic to rat NSCs. ${ }^{25}$ The effects of chemotherapy on human NSCs are unknown.

METHODS Standard protocol approvals, registrations, and patient consents. IRB approval was obtained at University of California Irvine Medical Center and Children's Hospital of Orange County.

Isolation and expansion of cells. NSCs (SC23, SC27, SC30) were derived from brains of premature neonates and cultured as previously described. ${ }^{26} \mathrm{HuTuP01}$ glioblastoma multiforme GSCs were a gift from Dr. David Panchision (Children's National Medical Center), ${ }^{27}$ and the stable tumor cell lines D54-MG and U251 were gifts from Dr. Darrell Bigner (Duke). Fresh brain tumors (low- and high-grade) were taken at surgery and dissociated, with neuropathologic review completed by a specialty neuropathologist (table e-1 on the Neurology ${ }^{\circledR}$ Web site at www.neurology.org). Cells were cultured on matrigel-coated dishes in 1:1 DMEM:F12 medium (Irvine Scientific), containing 10\% BIT9500 (Stem Cell Technologies), $292 \mu \mathrm{g} / \mathrm{mL}$ glutamine (Irvine Scientific), $40 \mathrm{ng} / \mathrm{mL}$ FGF, $20 \mathrm{ng} / \mathrm{mL}$ EGF, and $20 \mathrm{ng} / \mathrm{mL}$ PDGF. For expansion, one-half of this medium was replaced every other day, and the cultures were passaged every 7 days or when confluent using Nonenzymatic Cell Dissociation Solution (Sigma). All our GSCs are cultured as previously published, ${ }^{27}$ are able to form spheres when grown on nonadherent surfaces, and have high surface expression of CD133+ in normoxic condition $(20 \%-40 \%)$, similar to NSC. In addition, if the GSCs are grown under conditions favoring glial differentiation, they go on to express GFAP, and if they are grown under conditions favoring neural differentiation, they go on to express $\beta-3$ tubulin, confirming their multipotential nature.

Cell proliferation analysis. Cell proliferation rates were determined by BrdU incorporation. At 3 or 7 days after TMZ or CIS treatment, the cells were incubated with BrdU for 24 hours, and then fixed. Monoclonal anti-BrdU antibody was added, followed by horseradish peroxidase-conjugated secondary antibody. Tetramethylbenzidine conversion was measured at dual wavelengths of 450 and $540 \mathrm{~nm}$, using a Spectra Max 250 Plate Reader.

Cell death quantification. Necrotic cell death was determined by propidium iodide staining. ${ }^{28}$ Cells were collected, incubated with propidium iodide $(5 \mu \mathrm{g} / \mathrm{mL})$, and analyzed by flow cytometry.
Gene expression analysis. RNA was isolated using Trizol (Invitrogen), and cDNA was synthesized using the SuperScript ${ }^{\circledR}$ cDNA Synthesis Kit (Invitrogen). Serial PCR reactions (SYBR Green Master Mix, Qiagen) were conducted to quantify the levels of MGMT, MSH1, MLH2, ABCG2, and the proteasome subunits $\beta$ types 5, 6, and 7 (PSMB5, PSMB6, and PSMB7) using a BioRad C100 Cycler. The levels were normalized to actin. The specificity of the primers used (table e-2) was confirmed for every PCR run by dissociation curve analysis.

Apoptosis analysis. NSCs and GSCs were treated with BTZ or the vehicle control. Shortly after treatment, $50 \mu \mathrm{M}$ of caspase-3 inhibitor Z-VAD-FMK was added (Enzyme Systems). Caspase-3 activity was measured using a Fluorometric Assay Kit (MBL); $10^{6}$ cells were harvested 24 hours after treatment with BTZ (5 nM) and ZVAD $(50 \mu \mathrm{M})$, as indicated, pelleted, and resuspended in cell lysis buffer. A total of $200 \mu \mathrm{g}$ protein cell extract was incubated with $200 \mu \mathrm{M}$ DEVD-pNA. Spectrophotometric detection of EVD-pNA was performed at $380 \mathrm{~nm}$ excitation and $460 \mathrm{~nm}$ emission. The results are expressed as a percentage of control samples. The results are means \pm standard errors of 6 independent determinations.

Proteasome activity analysis. We measured 20 S proteasome chymotrypsin-like activity using the $20 \mathrm{~S}$ Proteasome Activity Kit (Millipore). Briefly, NSCs and GSCs treated with BTZ or the vehicle were harvested 4 hours after treatment, pelleted, and resuspended in cell lysis buffer. A total of $200 \mu \mathrm{g}$ protein cell extract (supernatant) was incubated with the Proteasome Substrate Suc-LLVY-AMC for 2 hours. The fluorescence was measured using a 380/460 $\mathrm{nm}$ filter set.

Immunocytochemistry. Cells were fixed in 10\% buffered formalin, pretreated in Antigen Retrieval Citra Buffer (Biogenex), and, after blockage of with $3 \%$ hydrogen peroxide, avidin-biotin complex immunoperoxidase reactions were performed using an EGFR antibody (Invitrogen). The reactions were followed by a biotinylated goat-antimouse immunoglobulin G secondary antibody and then an avidin-biotin peroxidase complex. The chromogen was diaminobenzidine for all reactions. Cells were counterstained with hematoxylin. A section of normal breast provided the positive controls.

Statistical analysis. Graphs and statistical analyses were prepared using Prism 3.03 (Graph Pad). All values were presented as mean \pm SEM. Significance was determined with simple paired $t$ tests or one-way analysis of variance.

RESULTS Human NSCs are more vulnerable to TMZ in vitro than either high-grade or low-grade GSCs. We treated 3 NSC cultures and multiple GSC cultures derived from both low- and high-grade glial tumors with TMZ with the goal of recreating in vitro the effects of this first-line treatment for low- and highgrade glial tumors. We determined that NSCs are much more sensitive than GSCs to therapeutically relevant doses of TMZ (with a 50\% reduction in the number of NSCs being present in the cultures 7 days after as low as $50 \mu \mathrm{M}$ TMZ treatment as compared with controls (figure 1A). GSCs treatment with similar TMZ doses did not have any effect on cell numbers. This is a robust observation, confirmed in 3 NSC cultures, as well as in 2 low-grade derived GSC 
A

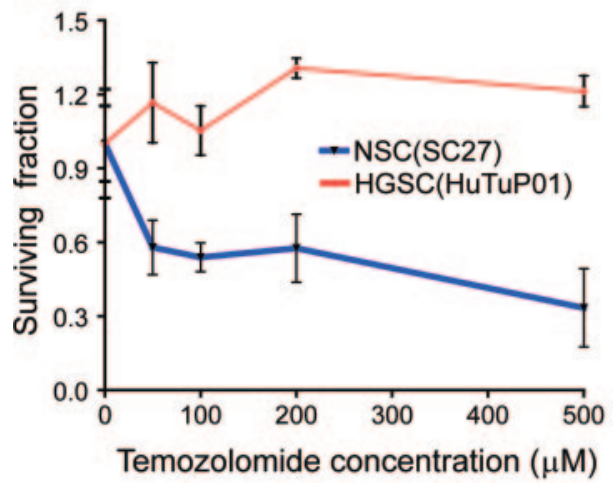

C

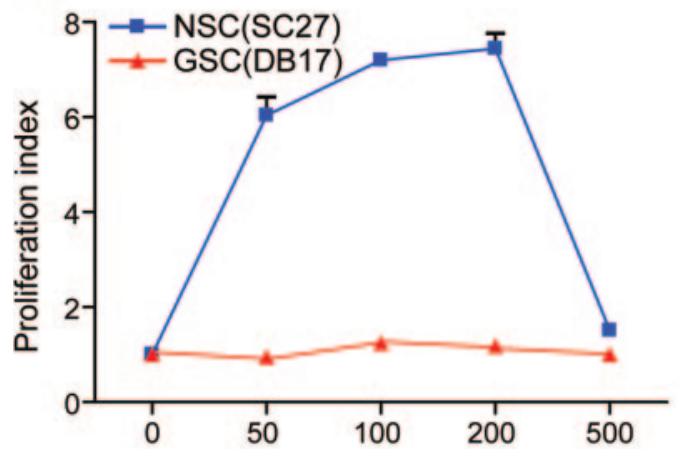

Temozolomide concentration $(\mu \mathrm{M})$
B

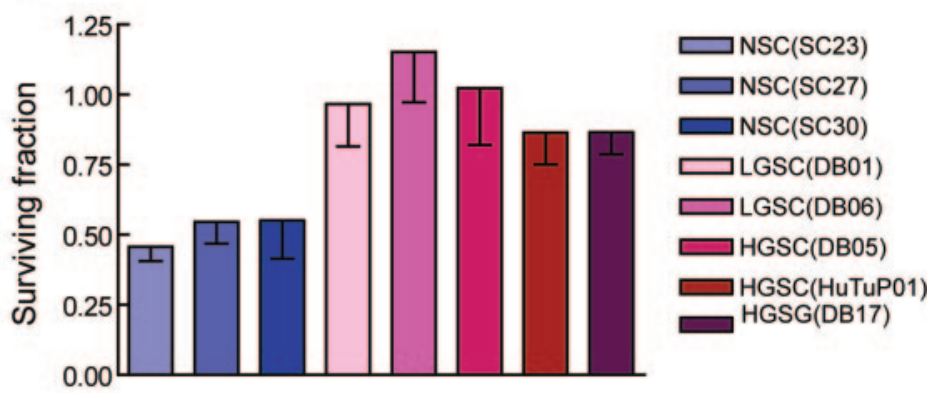

D

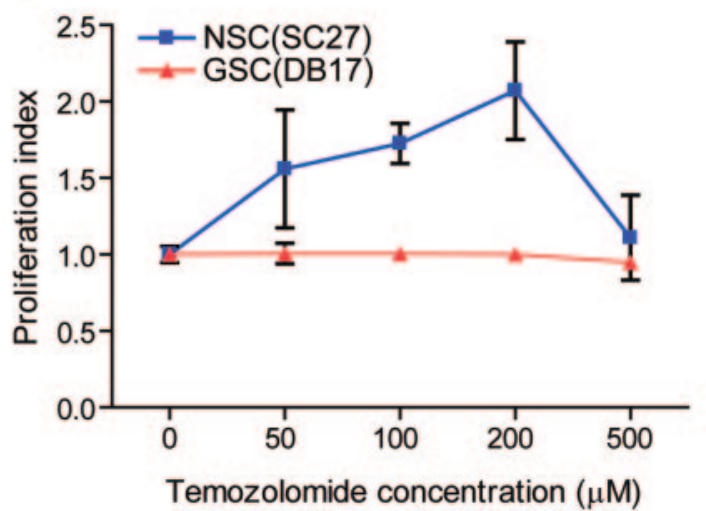

(A) In vitro treatment with graded doses of TMZ leads to a decreased number of NSCs (SC27) while minimally affecting GSCs (HuTuP01). (B) Treatment with $200 \mu \mathrm{M}$ of TMZ kills 50\% of the neural stem cells (SC23, SC27, and SC30). The same dose has less toxicity for low-grade (DB01, DB06) and high-grade (DB05, DB17, HuTu) GSCs. (C) Cellular proliferation of NSC as measured by BrdU incorporation is up to 8 times higher 3 days after the treatment with low doses of TMZ, but returns to lower levels 7 days after treatment (D). Seven days after TMZ treatment, no significant effects on proliferation are seen in GSCs.

cultures and 3 high-grade glioma-derived GSC cultures (figure 1B).

Low-dose but not high-dose TMZ stimulates NSC proliferation, while the GSC proliferation is not affected by $\mathrm{TMZ}$ treatment regardless of the dosage. To investigate the factors causing the NSC decrease, we estimated cell proliferation from the rate of BrdU incorporation. Low-dose TMZ (50-200 $\mu \mathrm{M})$ greatly increased the proliferation rate of NSCs at 3 days after the treatment - up to 8 times higher than the proliferation rate of vehicle-treated cells. However, higher TMZ doses $(500 \mu \mathrm{M})$ caused a lesser increase-just $50 \%$ compared with the vehicle-treated cells (figure $1 \mathrm{C}$ ). The increase in the proliferation rate was time-limited: 7 days after TMZ treatment the NSC proliferation rate at its maximum was just twice as high as the baseline levels (figure 1D). TMZ treatment had only a minimal effect on GSCs, with a faint $25 \%$ increase in proliferation observed at day 3 after treatment and essentially baseline levels at day 7 (figure 1, C and D).
TMZ induces cell death in NSCs but not GSCs. A decline in cell number can be secondary either to increased cell death or decreased proliferation. As NSC but not GSC proliferation was increased by TMZ treatment, we decided to determine if TMZ induced GSC cell death by measuring propidium iodide incorporation as a marker of necrotic cell death (figure 2A). Interestingly, 7 days after TMZ treatment, $25 \%$ of the NSCs (SC23) treated with $500 \mu \mathrm{M} \mathrm{TMZ}$ and $17 \%$ of the NSCs treated with $200 \mu \mathrm{M}$ TMZ were positive for PI staining. PI incorporation in GSCs was identical for the 2 treatment groups and the control, confirming that this tumor cell population is resistant to TMZ.

NSC and GSC have high and similar levels of the TMZ-resistance enzyme MGMT. MGMT is the most common marker of resistance to TMZ; high MGMT levels predict poor response to TMZ. ${ }^{8}$ Therefore, we measured MGMT expression in 3 NSC cultures, 11 GSC cultures derived from high- and low-grade glio- 
Figure 2 Temozolomide (TMZ) induces cell death in neural stem/progenitor cells (NSCs) but not in glioma stem-like cells (GSCs) by a methyl-guanine methyl transferase (MGMT)-independent mechanism
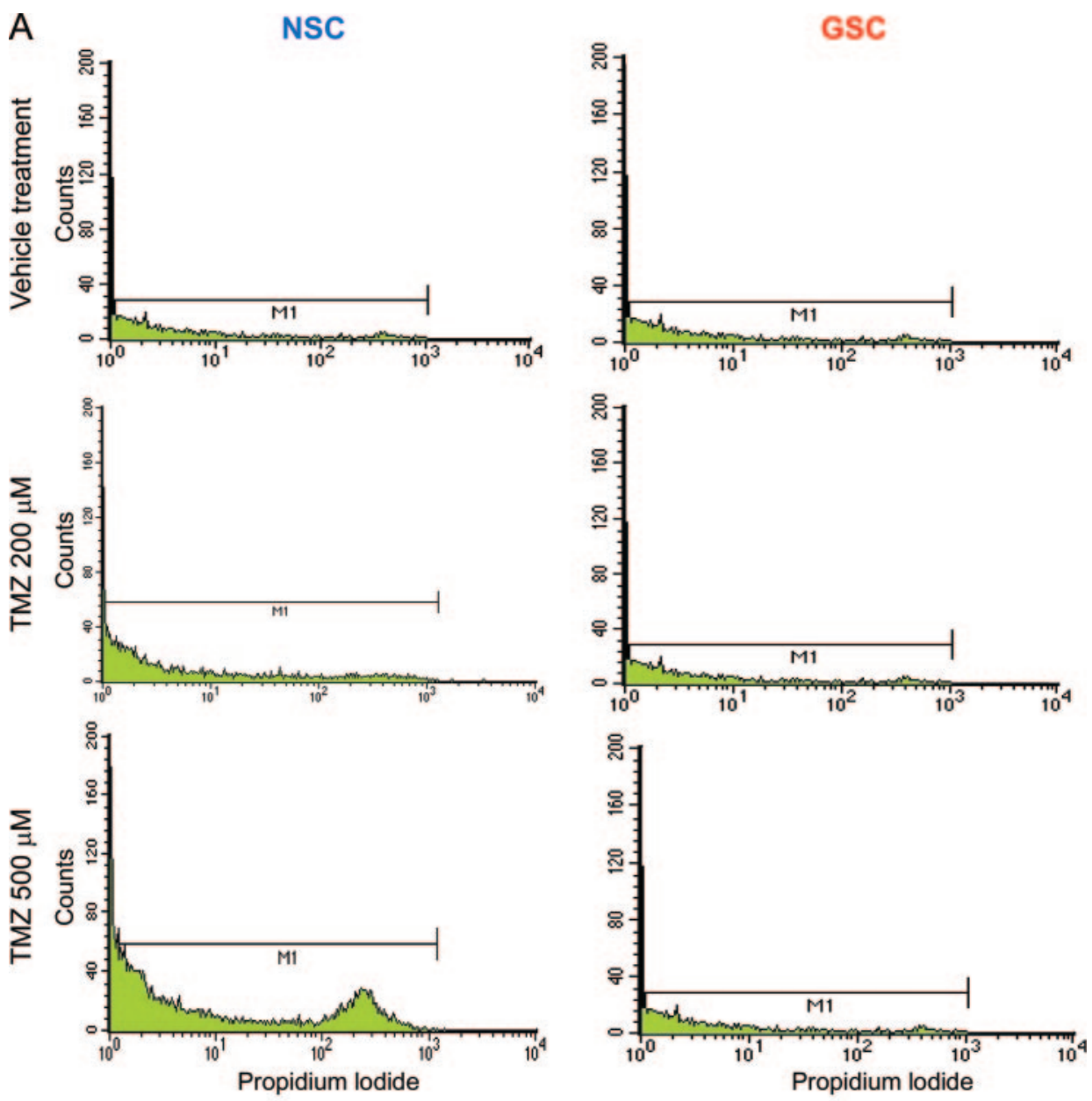

B

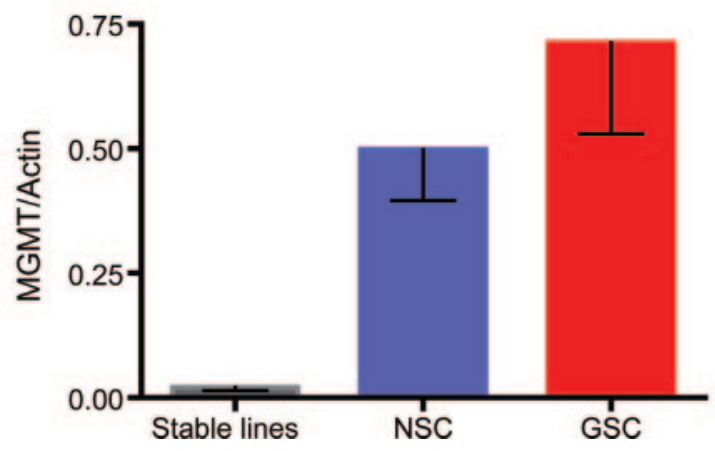

(A) Seven days after TMZ treatment, $25 \%$ of NSCs (SC23) treated with $500 \mu M$ TMZ and $17 \%$ of NSCs treated with 200 $\mu \mathrm{M}$ TMZ were positive for PI staining by flow cytometry, consistent with increased membrane permeability indicative of necrotic cell death, while GSCs (DB 17) were not affected. (B) NSCs and GSCs have similar gene expressions for the enzyme controlling resistance to TMZ (MGMT), but much higher expressions than the stable glioma cell lines. The data are average values of 3 NSC cultures, 11 GSC cultures, and the 2 stable glioma lines.

mas, and the 2 stable glioma cell lines. The results show that both NSCs and GSCs have very high and similar MGMT expression when compared with the stable cell lines, suggesting that differences in MGMT expression do not explain the observed differences in the response to TMZ (figure 2B).
Human NSCs are more vulnerable to CIS in vitro than GSCs. CIS is very commonly used as a second-line treatment for glioma and first-line chemotherapy for childhood CNS malignancies (e.g., medulloblastoma). We treated 2 NSC cultures and multiple GSC cultures with CIS (representative data shown). 
A

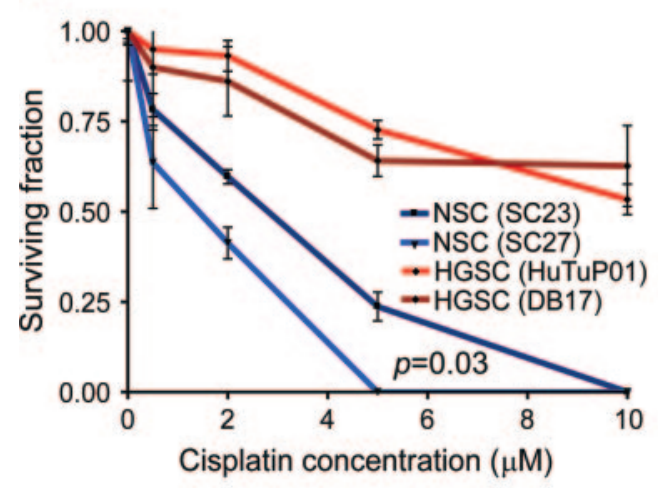

C

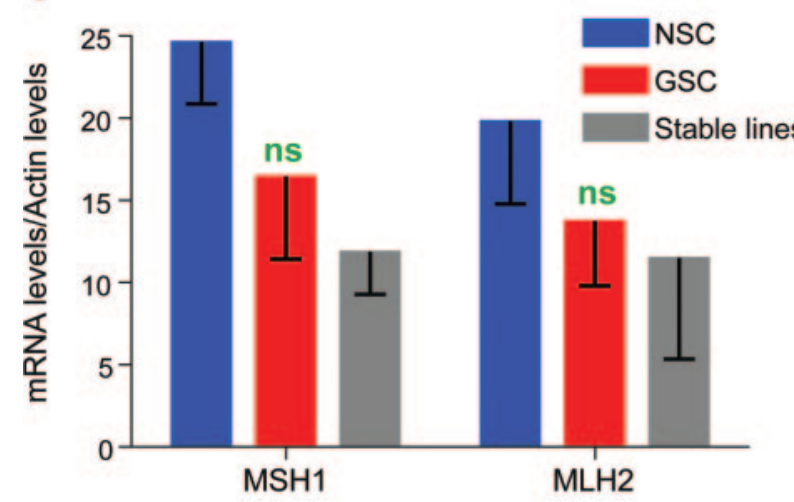

B

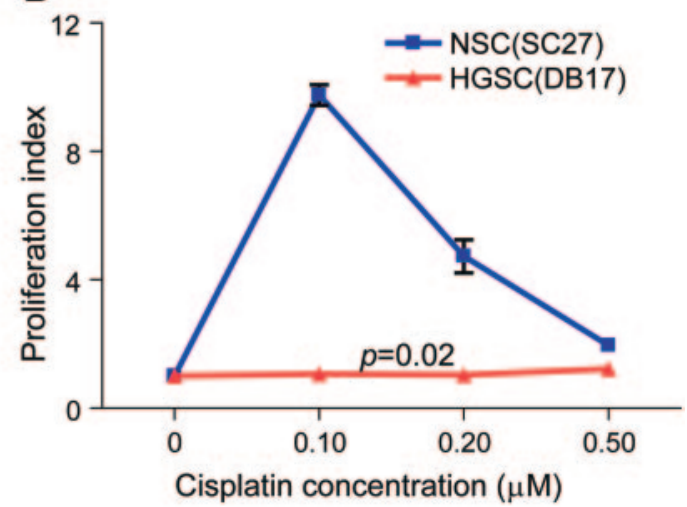

D

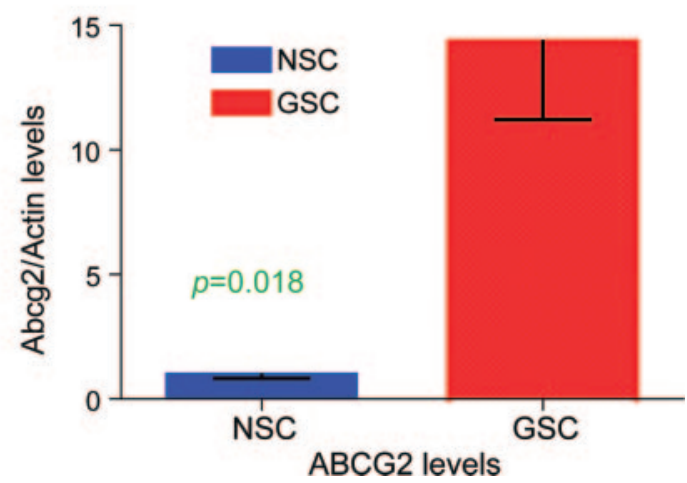

(A) In vitro treatment with graded doses of CIS leads to a decreased number of NSCs (SC23, SC27) while minimally affecting GSCs (HuTu, DB17). (B) Cellular proliferation of NSCs, as measured by BrdU incorporation, is up to 10 times higher 3 days after the treatment with low doses of CIS, but returns to lower levels 7 days after treatment (data not shown). (C) NSCs and GSCs have similar gene expressions for the mismatch repair enzymes controlling resistance to $\mathrm{CIS}$ (MSH1, MLH2). (D) NSCs have significantly lower expression of the ABCG2 multidrug resistance gene. The data are average values of 3 NSC cultures, 11 GSC cultures, and the 2 stable glioma lines.

CIS had severe effects on NSCs-even a very low dose of CIS $(0.5 \mu \mathrm{M})$ led to a $50 \%$ decrease in the number of cells in the culture 3 days after the treatment, while doses as high as $5 \mu \mathrm{M}$ effectively eradicated the NSCs (figure 3A). In contrast, low doses of CIS did not decrease GSCs number, and high doses $(10 \mu \mathrm{M})$ resulted in less than a $50 \%$ decrease, strongly suggesting that GSCs are resistant to CIS in our system (figure 3A).

Only very low-dose CIS stimulates NSC proliferation, while GSC proliferation is not affected by CIS treatment regardless of dosage. Very low-dose CIS treatment $(0.1-0.2 \mu \mathrm{M})$ greatly increases NSC proliferation rate 3 days after the treatment, up to 10 times higher than the proliferation rate of vehicle-treated cells. However, low-dose CIS $(0.5 \mu \mathrm{M})$ resulted in only a $50 \%$ increase (figure $3 \mathrm{~B}$ ). The increase in the proliferation rate was again time-limited: 7 days after the CIS treatment, the NSC proliferation rate returned to baseline levels (data not shown). Both low- and high-dose CIS had minimal effect on GSCs, with no measurable increase in proliferation at either day 3 or day 7 after treatment (figure 3B).

NSCs and GSCs have similar gene expression levels for the mismatch repair enzymes MLH1 and MSH2, but GSCs have higher levels for the multidrug resistance protein ABCG2. Sensitivity to CIS is governed by multiple mechanisms, including the presence of mismatch repair enzymes (MLH1, MSH2), and the ability of cells to extrude toxic drugs (ABCG2). Therefore, we measured MLH1, MSH2, and ABCG2 expression in 3 NSC cultures, 11 GSC cultures, and the 2 stable glioma cell lines. NSCs had higher expression levels of MLH1 and MSH2 than the stable lines $(p=0.048)$, but there was no difference between GSCs and NSCs (figure 3C). In contrast, ABCG2 expression levels were 10 times higher in GSCs than in NSCs $(p=0.018)$, suggesting that the higher resistance of GSCs to CIS and TMZ, as compared to NSCs, may be due 
Figure $4 \quad$ Neural stem/progenitor cells (NSCs) are less sensitive to proteasome inhibition than glioma stem-like cells (GSCs) due to higher proteasome expression and function and lower caspase-3 activation

A

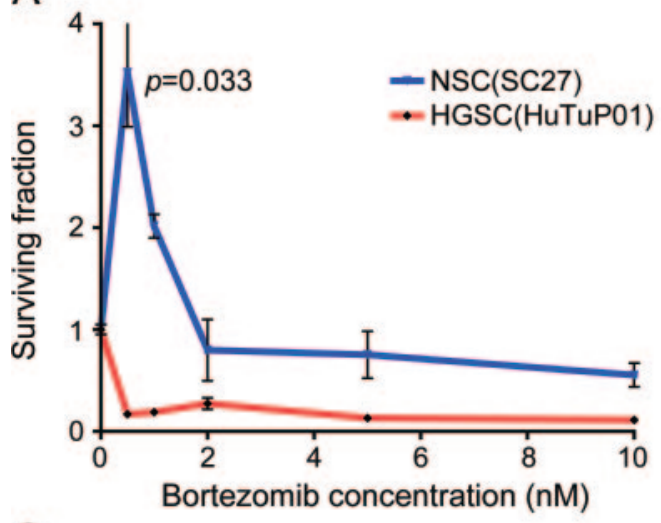

C
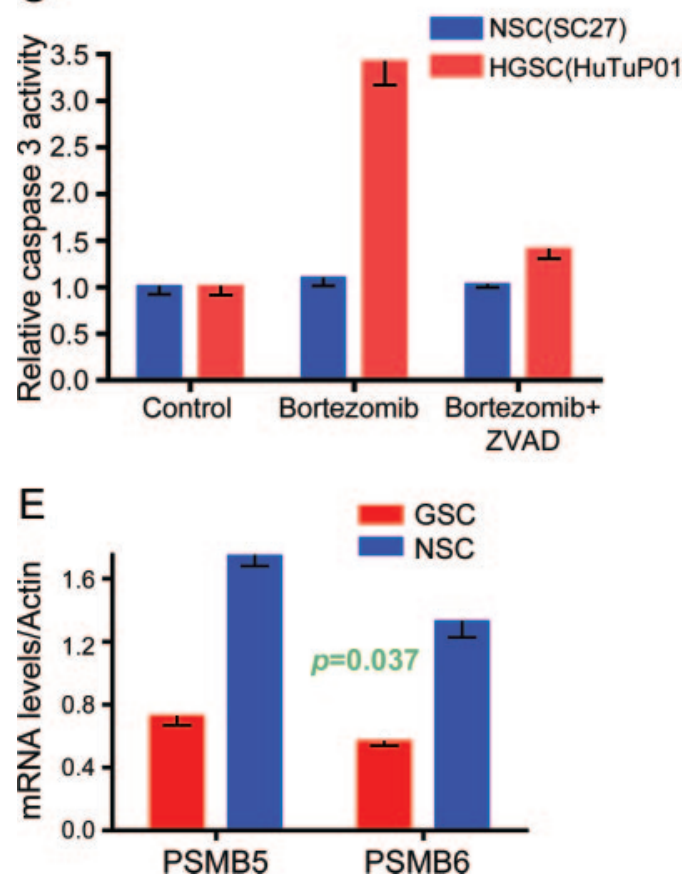

B

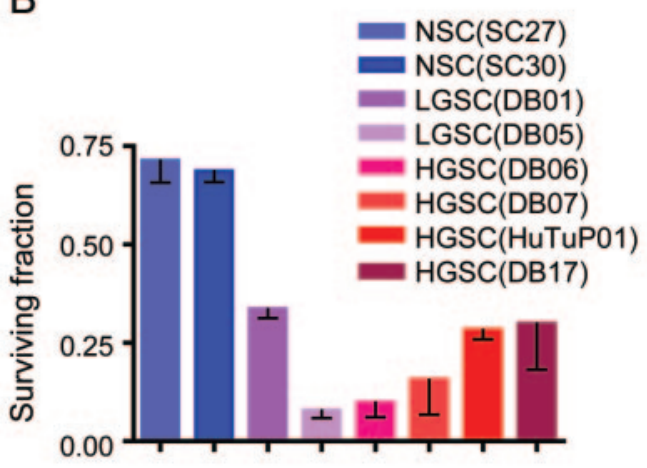

D

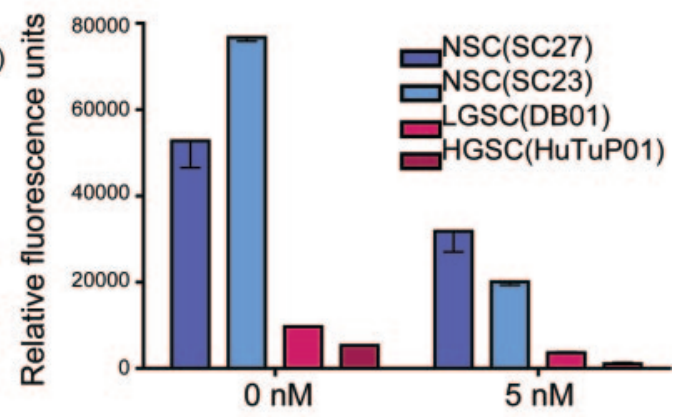

Bortezomib concentration

$\mathrm{F}$

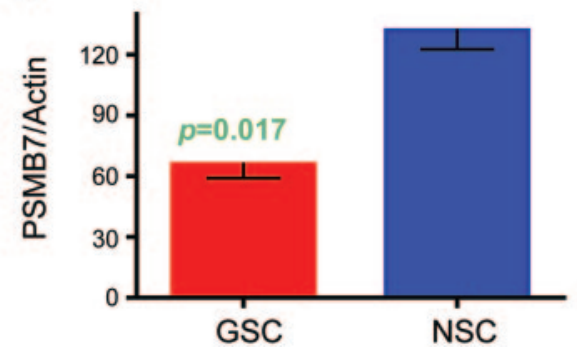

(A) In vitro treatment with graded doses of bortezomib (BTZ) kills off the GSCs (HuTuP01) while minimally affecting NSCs (SC27). (B) Treatment with $5 \mathrm{nM}$ of BTZ killed 75\%-90\% of the low-grade (DB01, DB06) and high-grade (DB05, DB07, DB17, HuTu) GSCs. The same dose had less toxicity for NSCs (SC27 and SC30). (C) Caspase-3 activity was measured in cell extracts, 24 hours after treatment with BTZ $(5 \mathrm{nM})$ and ZVAD $(50 \mu \mathrm{M})$, as indicated. GSCs had high levels of caspase-3 activation after the BTZ treatment, which was reversed by ZVAD addition, while the caspase-3 levels in NSC did not increase after BTZ treatment. (D) Proteasome activity was measured in cell extracts, at baseline and 4 hours after treatment with BTZ (5 nM). NSCs had higher baseline proteasome levels than GSCs, which remained higher than the GSC levels after the proteasome inhibition. (E, F) NSCs had significantly higher expression of the proteasome subunits PSMB5, PSMB6, and PSMB7. The data are average values of 3 NSC cultures, 11 GSC cultures, and the 2 stable glioma lines.

to greater GSCs ability to extrude chemotherapy drugs (figure 3D).

NSCs are less sensitive to proteasome inhibition than GSCs. To examine whether other chemotherapy agents could eradicate GSCs while preserving NSCs, we tested the sensitivity of the 2 groups of cells to proteasome inhibition with BTZ. The results show that GSCs were very sensitive to BTZ, with doses as low as $0.5 \mathrm{nM}$ causing more than an $80 \%$ reduction in cell numbers 3 days after the treatment (figure 4A). Treatment with similar low doses of BTZ (0.5-1 nM) stimulated NSC growth, with cell numbers $2-3$ times higher in the treatment group than in the vehicle control, while doses as high as $5 \mathrm{nM}$ minimally decreased the cell numbers as compared with the controls. The results were confirmed in 2 NSC cultures, 2 low-grade GSC cultures, and 4 high-grade GSC cultures (figure 4B).

BTZ induces caspase-3 activation in GSCs but not in NSCs. To examine the causes of observed reduction in cell numbers and cell death in the GSCs treated 
Figure 5 EGFR pathway inhibition with ERL preferentially affects glioma stem-like cells (GSCs) and correlates with EGFR expression
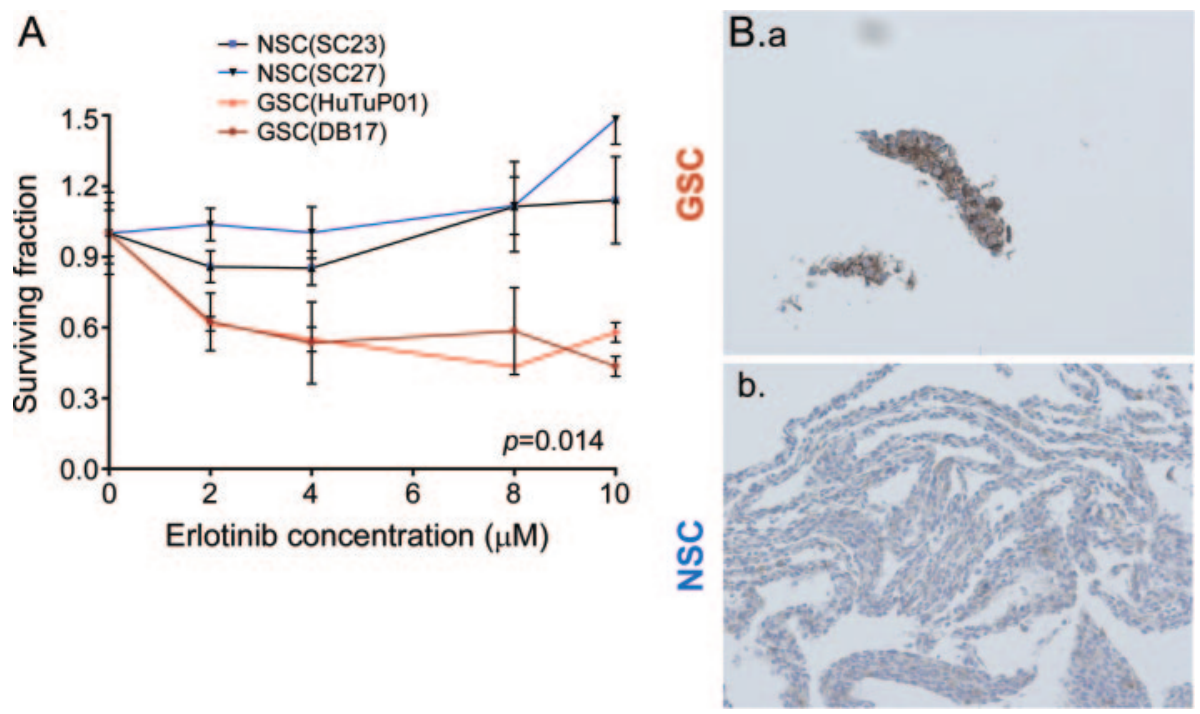

(A) In vitro treatment with graded doses of ERL led to a decreased number of GSCs (HuTu, DB17) while minimally affecting neural stem/progenitor cells (NSCs) (SC23, SC27). (B.a) GSCs are overwhelmingly positive for EGFR staining while (B.b) NSCs have low and sporadic expression (3 NSCs cultures and 5 GSCs cultures tested, representative results shown).

with BTZ, we measured the activity of caspase-3, a key enzyme involved in the early stages of apoptosis, which has been shown to be a major mediator of proteosome inhibitor toxicity in myeloma cell lines. ${ }^{29}$ Treatment with $5 \mathrm{nM} \mathrm{BTZ} \mathrm{resulted} \mathrm{in} \mathrm{a} \mathrm{vigorous}$ increase in caspase-3 activity, while addition of caspase-3 inhibitor Z-VAD-FMK effectively decreased caspase-3 activity in GSCs (figure 4C). No caspase-3 induction was seen in the BTZ-treated NSC, suggesting that NSCs were resistant to proteasome inhibition.

NSCs have higher proteasome activity and higher levels of PSMB5, PSMB6, and PSMB7 than GSCs. The cellular factors conferring resistance to BTZ are a subject of active research, but a number of parameters have been identified, such as increased proteasome activity in the resistant cells and mutations/ overexpression of proteasome subunits, especially PSMB5. ${ }^{30}$ We found that NSCs have 5-7 times higher proteasome chymotrypsin-like baseline activity than the low-grade and high-grade GSCs. We also discovered that, after BTZ-induced proteasome inhibition, NSCs still retained high levels of proteosome activity (up to 10 times higher than that seen in the treated GSCs; figure 4D). We measured PSMB5, PSMB6, and PSMB7 levels in 3 NSC cultures and 11 GSC cultures derived from high- and low-grade gliomas. On average, the levels of all 3 subunits were higher in NSCs than in GSCs, correlating with their high proteasome functional activity (figure 4, E and F).
EGFR pathway inhibition with ERL preferentially affects GSCs and correlates with EGFR expression. The EGFR tyrosine kinase inhibitor ERL has been actively studied as a targeted drug in glioma trials. ${ }^{11,12}$ In our experiments, treatment with neither low (2 $\mu \mathrm{M})$ nor high $(10 \mu \mathrm{M})$ dose of ERL affected the number of surviving NSCs, but both decreased the number of GSCs up to $50 \%$ (figure $5 \mathrm{~A}$ ). The response to ERL correlated with EGFR expression seen by immunocytochemistry: GSCs had consistently high expression of EGFR, while NSCs had only sporadic, low-level expression (figure 5B).

DISCUSSION The effects of chemotherapy on NSC survival depend on the type of chemotherapy studied (table e-3). The DNA-targeting drugs-namely TMZ and CIS-severely and preferentially kill NSCs compared to GSCs. This reduction in the number of NSCs (CIS can effectively eliminate the NSCs in our experimental conditions) may explain the cognitive impairments seen in brain tumor patients after treatment with these drugs. Unfortunately, neither TMZ nor CIS effectively eliminate GSCs, an observation which confirms previous studies suggesting that GSCs are resistant to chemotherapy $^{20}$ and could account for the limited prolonged survival by these treatments. ${ }^{31}$

In contrast to TMZ and CIS (which target equally the DNA of cancer and normal cells), the proteasome inhibitor BTZ is more toxic to GSCs, due to the relatively low functional level of the proteasome in GSCs. 
Even in low concentrations, this drug nearly eliminates GSCs. Even though BTZ has low penetrance in the CNS, these data might explain why BTZ is effective in reducing intracerebral tumor volume in mouse models of medulloblastoma ${ }^{32}$ as well as in a phase I study of recurrent malignant glioma. ${ }^{33}$

Malignant gliomas are characterized by EGFR overexpression, which is present in $40 \%$ to $60 \%$ of high-grade tumors. ${ }^{34}$ As ERL also acts at the protein level, by specifically binding the phosphorylation site of the receptor and prohibiting EGFR autoactivation, ERL treatment does not decrease the number of NSCs, but is able to target (though not very effectively) the GSC. This might occur because NSCs express surface EGFR only at certain developmental stages, ${ }^{35}$ while GSCs express EGFR consistently.

Treatment of brain malignancies with radiation and chemotherapy frequently results in learning and memory deficits in survivors. ${ }^{2,3}$ Radiation reduces hippocampal neurogenesis, ${ }^{36}$ resulting in a relatively diffuse pattern of cognitive impairment, involving multiple cognitive domains. ${ }^{37}$ The cognitive effects of chemotherapeutic agents, as distinct from radiation therapy, are harder to measure, as brain tumor patients usually receive both, and single or multiple chemotherapy agents are used at different disease stages. However, adult patients with primary CNS lymphoma who have received chemotherapy without radiation (high-dose methotrexate) still develop a decline in their attention, learning, and memory, ${ }^{37}$ which is more pronounced that the reported effects of chemotherapy in patients with breast and systemic lymphoma. $^{5}$ The late effects of chemotherapy (even in absence of radiation) for childhood brain tumors are prolonged and severe, with many persons exhibiting a developmental delay. ${ }^{38}$ Since pediatric patients receive either cisplatin or carboplatin, ${ }^{38}$ it is of interest that these agents result in massive NSC death, which might explain the patient's pervasive cognitive loss.

Our data might also explain why the malignant glioma tumors respond to therapy for a given time, and then recur. The 5-year follow-up for patients with newly diagnosed GBM treated with combined radiation and TMZ shows that fewer then $10 \%$ of the patients survive. ${ }^{39}$ If GSCs are resistant to TMZ, then GSCs will continuously repopulate the tumor, giving rise to daughter cells that may develop TMZ resistance. As ERL and BTZ seem to be more effective against the GSCs, these drugs could be targeted in future clinical trials as maintenance therapy after TMZ. However, the drug that is most effective against GSC_BTZ_ - has limited brain penetrance and considerable systemic toxicities, so development of new proteasome inhibitors would be of benefit. While ERL does not affect NSCs, it only decreases
GSCs survival by $50 \%$. This finding might explain the clinical data suggesting that ERL has very limited clinical activity as a single agent. ${ }^{40}$ Insufficient penetrance in the tumor and possibly the emergence of more differentiated, precursor-like glioma cells resistant to the EGFR blockage are alternative potential explanations.

Commonly, patients receive chemotherapy for many years in combination with other CNS-targeted treatments. Although the clinical focus should be on achieving remission (and possibly a cure), it is also important to attempt to maintain the quality of life of the survivors by avoiding severe long-term side effects. Using cancer drugs that have the potential to eradicate the most aggressive glioma stem-like cells, but preserve neural stem/progenitor cell populations and brain plasticity, might move us closer to that goal.

\section{ACKNOWLEDGMENT}

The authors thank Dr. Richard Kim for neuropathologic diagnosis of our tumor samples, Kelsey Albert, MS, and Ziren Research for technical assistance, and Dr. Tallie Z. Baram for constructive advice on the manuscript. The immunocytochemistry was completed with the help of the Pathology Services Core at UC Irvine.

\section{DISCLOSURE}

Dr. Gong reports no disclosures. Dr. Schwartz has received research support from Children's Hospital of Orange County (CHOC), the CHOC Foundation for Children, Autism Speaks, and the NIH. Dr. Linskey serves on a scientific advisory board for and has received funding for travel from Epitopoietic Research Corporation; is a member of the Medical Advisory Board and Co-Deputy Director for TNA-The Facial Pain Association; serves on the editorial board of Journal of Neuro-oncology; has a patent pending re: Endovascular optical coherence tomography device; and has received research support from a philanthropic patient. Dr. Bota serves on a scientific advisory board for the Epitopoietic Research Corporation; serves on speakers' bureaus for Merck Serono, Roche, and Eisai Inc.; serves on editorial advisory boards for Cancer Growth and Metastasis and Clinical Medicine Reviews in Oncology; and has received research support from American Cancer Society.

Received May 20, 2010. Accepted in final form October 15, 2010.

\section{REFERENCES}

1. 2010 CBTRUS Statistical Report: Primary brain and central nervous system tumors diagnosed in the United States in 2004-2006. In: Central Brain Tumor Registry of the United States. Available at: http://www.cbtrus.org/reports/ reports.html. Accessed March 2010.

2. Schmidinger M, Linzmayer L, Becherer A, et al. Psychometric and quality-of-life assessment in long-term glioblastoma survivors. J Neurooncol 2003;63:55-61.

3. Imperato JP, Paleologos NA, Vick NA. Effects of treatment on long-term survivors with malignant astrocytomas. Ann Neurol 1990;28:818-822.

4. Corn BW, Wang M, Fox S, et al. Health related quality of life and cognitive status in patients with glioblastoma multiforme receiving escalating doses of conformal three dimensional radiation on RTOG 98-03. J Neurooncol 2009;95:247-257.

5. Ahles TA, Saykin AJ, Furstenberg CT, et al. Neuropsychologic impact of standard-dose systemic chemotherapy in 
long-term survivors of breast cancer and lymphoma. J Clin Oncol 2002;20:485-493.

6. Armand JP, Macquet JP, LeRoy AF. Cerebrospinal fluidplatinum kinetics of cisplatin in man. Cancer Treat Rep 1983;67:1035-1037.

7. Ostermann S, Csajka C, Buclin T, et al. Plasma and cerebrospinal fluid population pharmacokinetics of temozolomide in malignant glioma patients. Clin Cancer Res 2004; 10:3728-3736.

8. Hegi ME, Diserens AC, Gorlia T, et al. MGMT gene silencing and benefit from temozolomide in glioblastoma. N Engl J Med 2005;352:997-1003.

9. Kartalou M, Essigmann JM. Mechanisms of resistance to cisplatin. Mutat Res 2001;478:23-43.

10. Diestra JE, Scheffer GL, Català I, et al. Frequent expression of the multi-drug resistance-associated protein BCRP/ MXR/ABCP/ABCG2 in human tumours detected by the BXP-21 monoclonal antibody in paraffin-embedded material. J Pathol 2002;198:213-219.

11. Haas-Kogan DA, Prados MD, Tihan T, et al. Epidermal growth factor receptor, protein kinase B/Akt, and glioma response to erlotinib. J Natl Cancer Inst 2005;97:880-887.

12. Rich JN, Reardon DA, Peery T, et al. Phase II trial of gefitinib in recurrent glioblastoma. J Clin Oncol 2004;22:133-142.

13. Gregory JK, Maria W-W, Mitchell M, et al. Phase I trial using proteasome inhibitor bortezomib and concurrent temozolomide and radiotherapy for central nervous system malignancies. Int J Radiat Oncol Biol Phys 2009;74:433-439.

14. Yin D, Zhou H, Kumagai T, et al. Proteasome inhibitor PS341 causes cell growth arrest and apoptosis in human glioblastoma multiforme (GBM). Oncogene 2004;24:344-354.

15. Sarkaria JN, Carlson BL, Schroeder MA, et al. Use of an orthotopic xenograft model for assessing the effect of epidermal growth factor receptor amplification on glioblastoma radiation response. Clin Cancer Res 2006;12:2264-2271.

16. Bao $S, W u$ Q, Sathornsumetee $S$, et al. Stem cell-like glioma cells promote tumor angiogenesis through vascular endothelial growth factor. Cancer Res 2006;66:7843-7848.

17. Singh SK, Clarke ID, Terasaki M, et al. Identification of a cancer stem cell in human brain tumors. Cancer Res 2003; 63:5821-5828.

18. Singh SK, Hawkins C, Clarke ID, et al. Identification of human brain tumour initiating cells. Nature 2004;432:396-401.

19. Bao S, Wu Q, McLendon RE, et al. Glioma stem cells promote radioresistance by preferential activation of the DNA damage response. Nature 2006;444:756-760.

20. Liu G, Yuan X, Zeng Z, et al. Analysis of gene expression and chemoresistance of CD133+ cancer stem cells in glioblastoma. Molecular Cancer 2006;5:67.

21. Reynolds B, Weiss S. Generation of neurons and astrocytes from isolated cells of the adult mammalian central nervous system. Science 1992;255:1707-1710.

22. Saxe MD, Battaglia F, Wang J-W, et al. Ablation of hippocampal neurogenesis impairs contextual fear conditioning and synaptic plasticity in the dentate gyrus. Proc Natl Acad Sci 2006;103:17501-17506.

23. Trouche SP, Bontempi B, Roullet P, Rampon C. Recruitment of adult-generated neurons into functional hippocampal networks contributes to updating and strengthening of spatial memory. Proc Natl Acad Sci 2009;106:5919-5924.

24. Monje ML, Mizumatsu S, Fike JR, Palmer TD. Irradiation induces neural precursor-cell dysfunction. Nat Med 2002;8:955-962.
25. Seigers R, Schagen SB, Beerling W, et al. Long-lasting suppression of hippocampal cell proliferation and impaired cognitive performance by methotrexate in the rat. Behav Brain Res 2008;186:168-175.

26. Schwartz PH, Bryant PJ, Fuja TJ, Su H, O’Dowd DK, Klassen $\mathrm{H}$. Isolation and characterization of neural progenitor cells from post-mortem human cortex. J Neurosci Res 2003;74:838-851.

27. Pistollato F, Chen H-L, Rood BR, et al. Hypoxia and HIF1alpha repress the differentiative effects of BMPs in high-grade glioma. Stem Cells 2009;27:7-17.

28. Crompton T, Peitsch MC, MacDonald HR, Tschopp J. Propidium iodide staining correlates with the extent of DNA degradation in isolated nuclei. Biochem Biophys Res Commun 1992;183:532-537.

29. Ri M, Iida S, Nakashima T, et al. Bortezomib-resistant myeloma cell lines: a role for mutated PSMB5 in preventing the accumulation of unfolded proteins and fatal ER stress. Leukemia 2010;24:1506-1512.

30. Oerlemans R, Franke NE, Assaraf YG, et al. Molecular basis of bortezomib resistance: proteasome subunit $\beta 5$ (PSMB5) gene mutation and overexpression of PSMB5 protein. Blood 2008;112:2489-2499.

31. Stupp R, Mason WP, van den Bent MJ, et al. Radiotherapy plus concomitant and adjuvant temozolomide for glioblastoma. N Engl J Med 2005;352:987-996.

32. Samano A, Ohshima-Hosoyama S, Whitney T, et al. Functional evaluation of therapeutic response for a mouse model of medulloblastoma. Transgenic Res 2010;19:829840.

33. Phuphanich S, Supko J, Carson K, et al. Phase 1 clinical trial of bortezomib in adults with recurrent malignant glioma. J Neuro-Oncol Epub 2010 Mar 8.

34. Frederick L, Wang XY, Eley G, James CD. Diversity and frequency of epidermal growth factor receptor mutations in human glioblastomas. Cancer Res 2000;60: 1383-1387.

35. Burrows RC, Wancio D, Levitt P, Lillien L. Response diversity and the timing of progenitor cell maturation are regulated by developmental changes in EGFR expression in the cortex. Neuron 1997;19:251-267.

36. Monje M. Cranial radiation therapy and damage to hippocampal neurogenesis. Dev Disabil Res Rev 2008;14: $238-242$.

37. Correa DD, DeAngelis LM, Shi W, Thaler H, Glass A, Abrey LE. Cognitive functions in survivors of primary central nervous system lymphoma. Neurology 2004;62:548555.

38. Nomura Y, Yasumoto S, Yanai F, et al. Survival and late effects on development of patients with infantile brain tumor. Pediatr Int 2009;51:337-341.

39. Stupp R, Hegi ME, Mason WP, et al. Effects of radiotherapy with concomitant and adjuvant temozolomide versus radiotherapy alone on survival in glioblastoma in a randomised phase III study: 5-year analysis of the EORTCNCIC trial. Lancet Oncol 2009;10:459-466.

40. van den Bent MJ, Brandes AA, Rampling R, et al. Randomized phase II trial of erlotinib versus temozolomide or carmustine in recurrent glioblastoma: EORTC Brain Tumor Group Study 26034. J Clin Oncol 2009;27:12681274. 